\title{
Performance of the ATLAS primary vertex reconstruction algorithms
}

\section{Matt Zhang*}

The ATLAS collaboration; Univ. Illinois at Urbana-Champaign (US)

E-mail: mzhang60@ilinois.edu

The reconstruction of primary vertices in the busy, high pile up environment of the LHC is a challenging task. The challenges and novel methods developed by the ATLAS experiment to reconstruct vertices in such environments will be presented. Such advances in vertex seeding include methods taken from medical imagining, which allow for reconstruction of very nearby vertices will be highlighted. The performance of the current vertexing algorithms using early Run-2 data will be presented and compared to results from simulation.

38th International Conference on High Energy Physics 3-10 August 2016

Chicago, USA

${ }^{*}$ Speaker. 